\title{
Tendencia en la enseñanza de la Ingeniería Mecatrónica y su campo disciplinar.
}

\author{
Jose Antonio Aquino Robles ${ }^{1}$, Leonel Germán Corona ${ }^{2}$ y Juan Carlos \\ Trujillo C. ${ }^{3}$
}

\section{Resumen}

En este trabajo se explican algunos elementos básicos que deben ser pormenorizados al observar la tendencia de la enseñanza de la Ingeniería Mecatrónica y su campo disciplinar. Lo anterior se realiza mediante un bosquejo de su evolución desde el punto de vista educativo. Recordando que la Mecatrónica como concepto surge en Japón a mediados de la década de los sesenta del siglo XX. Sin embargo fue concebida para ser enseñada como área de la ingeniería posteriormente. Después de un periodo en el que se construyó sus fundamentos en los que actualmente se cimientan sus saberes propios, como rama de la ingeniería moderna.

Desde su concepción, la Mecatrónica creó nuevos paradigmas tecnológicos, con una marcada diferencia a los esquemas tradicionales establecidos en aquellos momentos. En ese contexto la enseñanza de la Mecatrónica requiere de ser concebida de forma diferente a la enseñanza convencional de la ingeniería. Por lo cual, en este trabajo de investigación mostraremos una particular ilustración de su desarrollo, además de explicar también como se ha provocado dicha evolución. Así mismo de su campo disciplinar, juntamente con un incipiente modelo pedagógico. Todos estos temas en contexto a la naturaleza interdisciplinar de la Mecatrónica.

Palabras Clave: Enseñanza de la Mecatrónica, tendencias de la Mecatrónica, interdisciplinariedad, campo disciplinar, modelo pedagógico.

Fecha de recepción: julio 2013 | Fecha de aceptación: octubre 2013

${ }^{1}$ Instituto Politécnico Nacional, Universitat Politecnica de Catalunya.

${ }^{2}$ Instituto Politécnico Nacional, México.

${ }^{3}$ Instituto Tecnológico de Orizaba, México. 


\section{Abstract}

This paper explains some basic elements that should be detailed when observing the trend of Mechatronic Engineering education and its disciplinary field. This is done by an outline of its evolution from an educational standpoint, recalling that the Mechatronics as a concept emerged in Japan in the mid-sixties of the twentieth century. However, it was designed to be taught as engineering subject later after a period in which its foundations were built to be a branch of modern engineering.

Since its conception, Mechatronics created new technological paradigms, with a marked difference to the traditional schemes established at that time. In this context, Mechatronics teaching requires to be designed differently from conventional engineering teaching. Therefore, in this research we will show a special illustration of its development, and also explain this evolution origin. Likewise, we will explain its disciplinary field, together with an incipient pedagogical model -all these issues in context to the interdisciplinary nature of Mechatronics.

Keywords: Mechatronic Teaching, Mechatronic trends, interdisciplinary, disciplinary field, pedagogical model. 


\section{Introducción}

En la actualidad (2013), los conocimientos tecnológicos, científicos, las habilidades necesarias para que un ingeniero diseñe (modele) y construya: una máquina, o un componente de ella; como puede ser un dispositivo o todo un proceso productivo automatizado, lo llegue a optimizar en algún sentido. Son muy distintos de los necesarios hace apenas cuarenta años. Aun cuando los conocimientos científicos fundamentales en la formación de un ingeniero (la matemática, la física, y en su caso la química y la biología) son básicamente los mismos, estos deberán ya dosificarse de forma diferente en la enseñanza de la ingeniería en esta época y más aún en una rama de la ingeniería de las características de la Mecatrónica (Aquino, Corona, Fernández, 2012b).

La diferencia patente y subyacente respecto a las épocas, es la disponibilidad actual de dispositivos digitales como los ordenadores industriales, los cuales son empleados para controlar grandes máquinas; diseñar y/o controlar procesos automatizados o diseñar y construir dispositivos o componentes de estos complicados artefactos.

Una máquina automatizada diseñada hace cuarenta años, tendría complicadas articulaciones y levas las cuales definen la relación de movimiento coordinado entre las diversas estaciones ó etapas de la misma. En la actualidad, esas relaciones se realizan gracias a algún software de control, el cual permite la gestión por medio de un ordenador, o un autómata, o un micro controlador o procesador digital de señales (hardware).

Un diseñador de un sistema electromecánico controlado por ordenador (Ingeniero Mecatrónico) no sólo necesita saber principios apropiados de diseño mecánico, sino también conocer el hardware y el software de control, además de los sensores adecuados a fin de medir variables de interés y también las tecnologías de accionamiento. (Hidráulico, neumático, eléctrico etc.) Asimismo también manejar las herramientas de diseño asistido por ordenador en todas estas áreas $\mathrm{CAD}^{4}$, juntamente con las herramientas de manufactura moderna $\mathrm{CAM}^{5}, \mathrm{CAE}^{6}$ las cuales hacen posible que un Ingeniero Mecatrónico pueda diseñar la integración de todas las tecnologías necesarias, hasta el punto que pueda emplearlas efectivamente (Aquino, Corona, Fernández 2011).

Respecto a lo anterior se puede manifestar en una primera aproximación que la Mecatrónica es una nueva y singular rama de la ingeniería que se ha ido consolidando a partir de sinergias tecnológicas; las cuales por razones propias de

\footnotetext{
${ }^{4}$ CAD -Diseño asistido por ordenador del inglés (Computer-aided design)

${ }^{5}$ CAM Manufactura asistida por ordenador del inglés (Computer-aided manufacturing )

${ }^{6}$ CAE Ingeniería Asistida por ordenador del inglés (Computer Aided Engineering)
} 
su origen estuvieron enfocadas hacia la innovación. En el siguiente segmento de esta investigación se discutirán algunas definiciones propuestas en la literatura técnica contemporánea.

Por antonomasia un Ingeniero Mecatrónico deberá ser un diseñador en la integración de sistemas (desde el punto de vista productivo-industrial). Es muy poco común en ésta época (desde la perspectiva de la integración de sistemas) el caso en el que todos los componentes de un sistema se diseñen desde cero para un proyecto productivo, industrial, científico e incluso académico.

Con mucha frecuencia, el Ingeniero Mecatrónico selecciona componentes y subsistemas, de manera paralela diseña en forma adecuada la integración del hardware y software según se necesite. A ese respecto es menester señalar que el diseño de dispositivos específicos con tecnología de vanguardia, se desarrolla en líneas de investigación científica o mejor aún de; investigación tecnológica en estudios de nivel graduado (postgrado). Por lo que a nivel pregrado (licenciatura), lo más trascendente desde el punto de vista educativo, en cuanto a la virtud en la habilitación y preparación del Ingeniero Mecatrónico es: la eficiente habilidad que tenga integrando y seleccionando componentes, subsistemas, dispositivos de la tecnología más apropiada para desarrollar máquinas y sistemas Mecatrónicos. De todo ello se hablará en el presente trabajo en el cual se analizará la tendencia en la enseñanza de ésta rama de la ingeniería moderna. (Calderón E., Forero G. C.A., Chio N 2011)

\section{Análisis teórico}

Al iniciar en este segmento, es necesario manifestar que una de las definiciones de Mecatrónica consiste en la integración sinérgica de cuatro campos de la ingeniería tradicional para el proceso de diseño a nivel de sistema. Estos cuatro campos son:

- Ingeniería mecánica, (de donde se toma el prefijo "meca")

- Ingeniería electrónica, (de donde se toma el sufijo"trónica")

- Ingeniería de cómputo. (informática)

- Ingeniería de control.

Empero Álvarez, Neff, Moya Chagoyén, Machado (2012) citan algunas definiciones basadas en la siguiente frase:

La Mecatrónica es la integración de la mecánica y la electrónica en una máquina o producto.

Sin embargo, aún con lo simple que resulta la expresión anterior, es con el 
transcurso del tiempo juntamente con la cultura organizacional que le dio origen, la que logra arraigar a la Mecatrónica como una nueva especialidad de la ingeniería, la cual incorpora otros elementos como: los sistemas informáticos, los desarrollos de la microelectrónica, la inteligencia artificial, la teoría de control y otros relacionados con tecnologías de vanguardia.

Al madurar primero como concepto y posteriormente como rama de la ingeniería, comenzó a dictarse cátedra de esta nueva especialidad, en un principio en las escuelas de ingeniería de nivel graduado y posteriormente a nivel pregrado en las principales universidades de los países más industrializados del mundo. Y como muchas veces sucede en el mundo tecnológico, después de surgir un concepto o un desarrollo tecnológico innovador y atrayente, llega posteriormente la ciencia tratando de pormenorizar dicho desarrollo, por lo que posterior a su surgimiento, emergen las siguientes definiciones tratando de explicar lo que es la Mecatrónica.

- La definición propuesta por: J.A. Rietdijk mencionada por Álvarez et al (2012): - "Es la combinación sinérgica de la ingeniería mecánica de precisión, de la electrónica, del control automático y de los sistemas para el diseño de productos y procesos".

- Por su parte también Álvarez et al (2012) menciona la definición de Craig como: "La integración sinérgica de sistemas físicos, electrónica, controles y ordenadores a través del proceso de diseño desde el mismo comienzo del tal".

Asimismo añade: La integración es el elemento clave y la complejidad ha sido transferida desde el mando de los sistemas mecánicos al dominio de los ordenadores, el software y la electrónica. Señala también que la Mecatrónica es producto de un desarrollo evolutivo del diseño que demanda una integración horizontal entre numerosas disciplinas de la ingeniería así como una integración vertical. Resumiendo Craig plantea que la Mecatrónica es la mejor práctica para la síntesis en ingeniería, respondiendo a las necesidades de la industria y de los seres humanos de ésta época.

A lo anterior podemos añadir lo que se manifestó: Aquino, Corona (pp 29-31, 2009a), al afirmar que la "Mecatrónica" significa un sinfín de cosas para mucha gente, pero cuando se le pide a algún discípulo de esta rama de la ingeniería que trate de dar una definición precisa, por lo general se refiere a un dibujo mostrado por Kevin Craig referido por Álvarez et al (2012) que consiste en cuatro círculos sobrepuestos mostrados en la Figura 1, en los cuales se aprecian los:

- Sistemas mecánicos,

- Sistemas electrónicos,

- sistemas de control, y la

- Informática.

En el centro, donde se traslapan todas las sinergias primigenias asociadas, se forma la sinergia global "Mecatrónica". Sin embargo la Mecatrónica representa 
más que la mecánica y electrónica interactuando. La Mecatrónica está implicada con casi todo proceso de diseño de cualquier sistema físico, donde se integran: sensores, controles, electrónica, y computadoras desde el mismo comienzo de dicho proceso (Aquino, Fernández, Cuervo 2010)

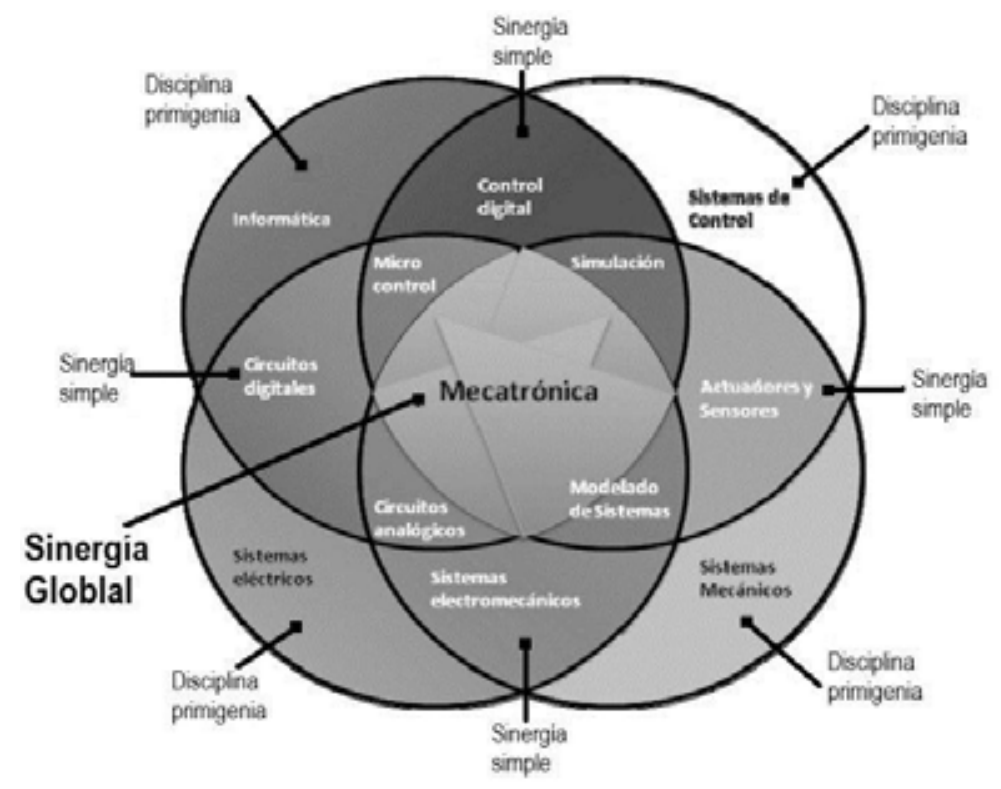

Figura 1. La conocida unión sinérgica de las áreas que conforman la ingeniería Mecatrónica.

(Álvarez et al 2012)

Respecto a lo anterior es válida la siguiente aclaración, puesto que es común creer que el Campo disciplinar ${ }^{7}$ de la Mecatrónica es la suma total de todas estas cuatro áreas de la ingeniería. Sí bien esta confusión podría ser producto de observar en un solo plano la figura 1, conocida por los estudiosos de la Mecatrónica, como la unión sinérgica de las cuatro áreas que la conforman. Existe sin embargo como elemento clave, la integración, siendo ésta tanto vertical como horizontal como menciona Craig.

Se señala también que la Mecatrónica implica un desarrollo evolutivo y un nuevo y definido campo disciplinar, empero este desarrollo evolutivo no ha surgido de forma espontánea, ni se da de manera azarosa, el tal es producto de la misma cultura organizacional que le dio origen a la Mecatrónica y además conlleva de forma implícita una singular filosofía de diseño ya muy bien definida en el espacio temporal.

${ }^{7}$ Campo disciplinar: Organización sistemática de una disciplina en particular para el logro del Perfil profesional y que se traduce en un plan de estudios. 
Lo anterior puede explicarse mejor observando la Figura 2, en la cual se aprecia (añadiendo la dimensión temporal-espacial) su identidad como un cuerpo disciplinar autónomo; además de su evolución tecnológica sustentándose en las áreas que la han precedido en lo temporal y la respaldan mediante la integración de tecnologías tanto en lo vertical como en lo horizontal en un marco técnico-científico (Aquino, Corona Fernández, 2011)

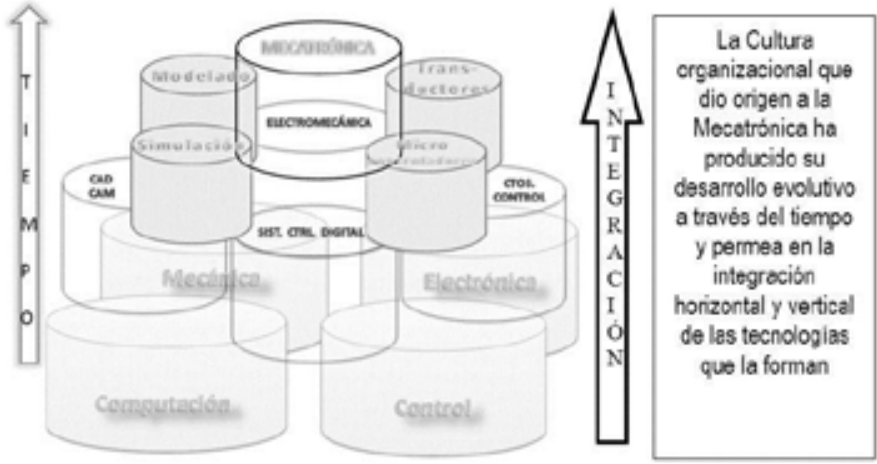

Figura 2. Evolución de los campos disciplinares que dieron origen a la Mecatrónica.

(Aquino et al 2011).

\section{Surgimiento como tecnología}

Si nos remontamos al sitio de origen de la Mecatrónica podemos manifestar que ésta germina en una nación con una prestigiosa cultura basada en la innovación como Japón (Aquino et al pp 29-31, 2009a). Dicha cultura organizacional ha permeado de tal forma que se ha logrado una amplia interacción entre disciplinas (interdisciplinariedad), misma que ha resultado ser pilar del desarrollo tecnológico de la era digital (Aquino et al pp 29-31, 2009b).

\section{Integración Horizontal}

Históricamente se sabe que de la sinergia entre las disciplinas primigenias surgió primeramente con la Ingeniería de control con el regulador de Watt en la máquina de Vapor; posteriormente La Electromecánica, surgida del enlace entre la ingeniería eléctrica y la mecánica, posteriormente, la informática y con ella los programas CAD y CAM, los sistemas de control digital, vea la Figura 3.

En algunos casos, estas sinergias han dado paso o son ya reconocidas áreas de la ingeniería, en otros casos son líneas de investigación y desarrollo tecnológico y en otros más, herramientas de ingeniería que surgieron anteriores en algunos casos a la de la sinergia global Mecatrónica (Aquino et al 2010).

Sí bien es cierto la informática surge inicialmente como medio o herramienta para hacer más rápido y con mayor precisión el cálculo en ingeniería, posteriormente se va descubriendo que ésta versátil herramienta puede mejorar en muchos aspectos, 
el trabajo y la forma de vida humana. Y su vertiginoso crecimiento la posiciona como objeto de investigación y desarrollo tecnológico, hasta el punto en que se le consideró y empezó a enseñarse ya de forma autónoma (Ingeniería en sistemas computacionales).

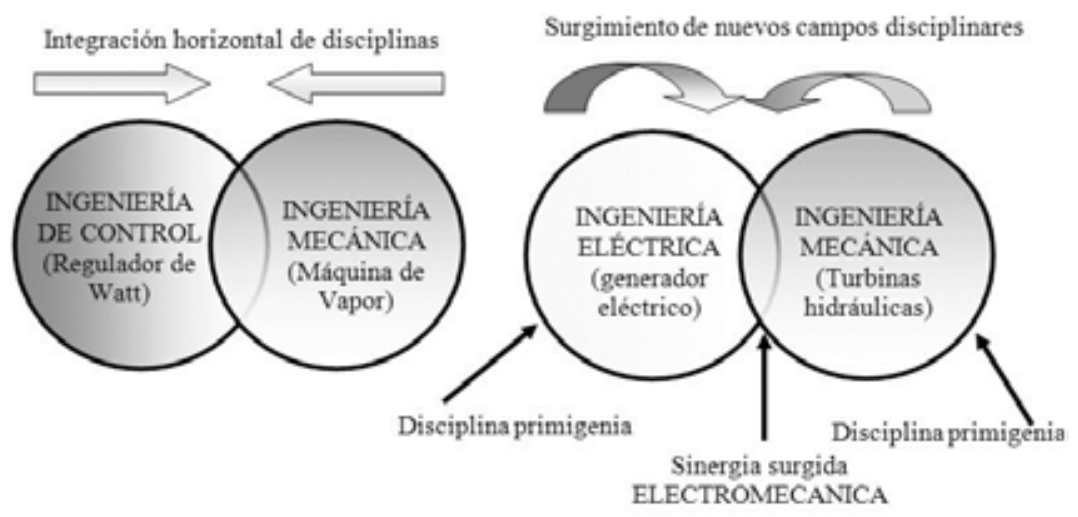

Figura 3. Integración horizontal de disciplinas de ingeniería

En el trabajo de Aquino, Cuervo, Corona, (pp 141-145, 2011) se menciona que la cultura organizacional japonesa, cuyo objetivo es la innovación mediante su singular forma de trabajo; produjo sinergias entre diversas ramas de la ingeniería y esta a su vez ha dejado tras de sí mayor interdisciplinariedad (enlace entre las disciplinas) y otras tantas más herramientas, (de ingeniería). Estas sinergias han ido trascendiendo más allá de su utilización original. Como ejemplo fehaciente está la necesidad de predecir el comportamiento de diferentes materiales a esfuerzos o solicitaciones mecánicas de forma rápida e incluso sin tener que construir un prototipo o maquinar probetas y hacer con estas, costosas pruebas, de ahí el uso de los métodos numéricos, que gracias a los lenguajes y programas informáticos juntamente con poderosos procesadores digitales han hecho posible las tareas de análisis, dando como resultado una línea de investigación y herramienta de ingeniería llamada modelado y simulación de sistemas y/o procesos y también el software CAD mecánico de análisis y de diseño.

\section{Integración vertical}

Antiguamente para el diseño de un proceso o máquina o dispositivo electromecánico se requería:

- Ingenieros que diseñarían los componentes mecánicos.

- Ingenieros que diseñarían los componentes eléctricos como actuadores, sensores y amplificadores, así como el diseño de la lógica y los algoritmos de control.

- Ingenieros que diseñarían la implementación del hardware y software de cómputo para controlar la producción en tiempo real. 
Sin embargo en la actualidad y después del proceso evolutivo producto de la mencionada cultura organizacional que dio origen a la Mecatrónica. El trabajo de diseño mencionado anteriormente; mismo que desarrollaban ingenieros de diversas especialidades para lograr un objetivo en común. Ahora lo puede realizar un Ingeniero Mecatrónico con ayuda de las modernas tecnologías que se han desarrollado a través de la misma Ingeniería y de la creación de conocimiento organizacional. Siendo estas dos las que produjeron la evolución de la Mecatrónica. (Aquino, Corona, Fernández, 2011)

La Ingeniería Mecatrónica esquematiza en si misma esa forma de trabajo o cultura organizacional que ha producido sinergias a través del tiempo, pero más importante aún, las condensa en el trabajo estructurado que deberá demostrar un Ingeniero Mecatrónico en la práctica de la ingeniería en el sector productivo (Aquino, Corona, Fernández, 2010). Siendo esto uno de los aspectos más trascendentes y fundamentales al momento de desentrañar la tendencia en la enseñanza de la Mecatrónica. Ahora bien dentro de los elementos que podría servir para guiar ésta tendencia en la docencia de la Mecatrónica está sintetizado en contestar la siguiente pregunta:

¿Cómo recrear la forma de trabajo que hizo evolucionar a la Mecatrónica a lo largo del plan de estudios?

En contexto con la pregunta anterior, Álvarez et al (2012) manifiesta que en la actualidad existen numerosos desafíos para la enseñanza de la misma. Pero sí partimos de que la Ingeniería es la profesión en la que el conocimiento de las ciencias matemáticas y naturales adquiridas mediante el estudio, la experiencia y la práctica, se emplea con buen juicio a fin de desarrollar modos en que se puedan utilizar, de manera óptima los materiales y las fuerzas de la naturaleza en beneficio de la humanidad, en el contexto de restricciones éticas, físicas, económicas, ambientales, humanas, políticas, legales y culturales. Y que la Práctica de la Ingeniería comprende el estudio de factibilidad técnico económica, investigación, desarrollo e innovación, diseño, proyecto, modelación, construcción, pruebas, optimización, evaluación, gerenciamiento, dirección y operación de todo tipo de componentes, equipos, máquinas, instalaciones, sistemas y procesos (Aquino, Corona, Fernández, 2012a). Con ello podemos entender que la ingeniería tiene una finalidad muy bien definida, y por lo tanto el plan de estudios deberá tener también una finalidad de la misma naturaleza.

Empero para responder a la pregunta podemos manifestar que el plan de estudios puede organizarse, no sólo centrado en asignaturas, como ha sido costumbre, sino que puede planificarse alrededor de núcleos superadores de los límites de disciplinas, centrados en temas, casos, problemas y/o proyectos; que en el caso de la 
ingeniería implicara la solución de estos vía proyectos integradores. Como variante de esta perspectiva se concibe el Curriculum Holístico. La palabra holístico proviene del término griego "holos" que significa "totalidad". Expresa lo que se toma entero con sus diferentes partes. La propiedad holística exhibida por los sistemas (modelo conceptual o lógico creado para representar a un objeto concreto) depende de la organización entre las partes, de un orden entre ellas. En dicho principio se argumenta que el holismo o sinergia es un paradigma de resurgimiento, basado en una rica tradición de muchas disciplinas. El holismo afirma la interdependencia inherente entre la teoría, la investigación y la práctica, en constante evolución. El holismo tiene sus raíces en la proposición de que el universo es una totalidad integrada y que todo está conectado. Esta proposición de integración y unidad está en oposición directa al paradigma de separación y fragmentación que predomina en el mundo contemporáneo (Castellanos Álvarez J., Lara Díaz L., Lapido Rodríguez M., Iglesias León M., Balbis Coll E., Navales Coll M., Álvarez González A. 2003)

El objetivo del Curriculum Holístico es preparar a los estudiantes para transitar en una carrera en la cual sus éxitos estén condicionados por el aprendizaje durante su vida, pensamiento crítico, toma de decisiones, trabajos en grupo, liderazgo y seguridad. La base del Curriculum Holístico es la identificación de las cualidades deseadas para los graduados y la enseñanza de las habilidades a través de él, mismas que se han citado anteriormente.

Entre las características de un curriculum Holístico, tenemos:

- El empleo de la estrategia basada en proyectos coordinados.

- El papel que desempeñan los profesores y posteriormente los estudiantes.

- Los estudiantes enseñan a otros los conceptos que dominan, por lo que estos aprenden unos de otros.

- La introducción de las habilidades deseadas, para los egresados, desde el primer año y su desarrollo a través del curriculum.

- Dichas habilidades se refuerzan y retroalimentan para conocer su dominio

- La interrelación entre los contenidos.

- La asignación de problemas transdisciplinarios durante el curriculum y por la discusión de sus soluciones a través de presentaciones orales y escritas.

- El consumo de tiempo y la comunicación que se establece entre todos para lo que habrá de impartirse (contenidos).

- La forma en que se realiza la evaluación.

- La estrategia que se sigue en la elevación del nivel de complejidad de las tareas asignadas.

- La manera en que los estudiantes aprenden a trabajar en equipo y a comunicarse.

- La exigencia que se solicita al estudiante cuando rinde informe acerca de los diseños de proyectos coordinados.

- Los procedimientos que utilizan los estudiantes al defender sus decisiones 
en las clases u otras actividades.

- La estructura, el proceso de aprendizaje se da por lazos de retroalimentación.

- El conocimiento de los estudiantes de la razón del por qué ellos aprenden.

- La viabilidad de aprender los conceptos trabajando con los proyectos.

- La pertenencia de los estudiantes por el trabajo de la facultad.

- El dominio de los estudiantes de la mayoría de las habilidades que la industria desea.

- La integración de los contenidos desde los primeros años a partir de los proyectos.

- La presentación de los trabajos en público, lo que requiere de la presencia de estudiantes de diferentes años.

- El sistema de control y de retroalimentación, el cual posibilita que el contenido del curriculum pueda ser modificado basándose en la comparación entre la actual y lo deseado.

- La forma en que la facultad entera participa en la reorganización del curriculum, porque la idea de mejorar nace de dentro de ella misma y toda ella participa.

En el Curriculum Holístico las habilidades deseadas para los egresados se introducen desde el primer año y se desarrollan a lo largo de la carrera. Se posibilita que se refuercen continuamente a través de los diseños de proyectos que se ejecutan a nivel de años, grupos e individualmente. (Castellanos Álvarez J et al 2003).

Sin embargo después de ver estas recomendaciones en lo general cabe por preguntarse también lo siguiente pero ya en lo específico (para la ingeniería Mecatrónica):

¿Cómo lograr un diseño adecuado del campo disciplinar para la enseñanza de la Mecatrónica? y que por medio de él, se logre conjuntar los conocimientos de Ingeniería Mecánica, Electrónica, Informática y Control automático pero más importante aún debido al origen y modo de trabajo que se tiene en Mecatrónica, es necesario preguntarse ¿cómo dotar de las habilidades y el saber hacer (know how) para la Práctica de la Ingeniería Mecatrónica, y que esto satisfaga las crecientes demandas del desarrollo industrial y las habilidades que se requieren para dicho especialista? (Aquino, Corona, Fernández 2012c).

Sí partimos de que un sistema Mecatrónico típico recoge datos del mundo con el que interactúa mediante sensores, los procesa y como salida, genera fuerzas y movimientos. Los subsistemas mecánicos son entonces los actuadores o extensiones de estos y a su vez están integrados con sensores, microprocesadores y controladores.

Los robots, las máquinas controladas digitalmente, los vehículos guiados automáticamente, las cámaras electrónicas, las máquinas de telefax y las fotocopiadoras pueden considerarse como productos Mecatrónicos en su caso.

En el sitio donde surgió la Mecatrónica al aplicar la forma de trabajo o cultura organizacional, cuyo medio fue la integración de tecnologías y cuyo fin fue la innovación; se obtuvieron ventajas importantes en el diseño de productos y sistemas, como son mayor 
flexibilidad, versatilidad, nivel de inteligencia de los productos, seguridad y confiabilidad así como un bajo consumo de energía (Aquino, Corona, Fernández 2012d).

Estas ventajas se traducen en un producto con más orientación hacia el usuario y que puede producirse rápidamente a un costo reducido, vea Figura 4.

También de acuerdo Álvarez et al 2012 y a su clasificación de los productos Mecatrónicos:

A finales de los años 70 del siglo XX, la sociedad Japonesa para la promoción de la industria de maquinaria (JSPMI) clasificó productos Mecatrónicos en cuatro clases:

Clase I:

Productos primariamente mecánicos con la electrónica incorporada para realzar o aumentar su funcionalidad, por ejemplo máquinas herramientas con control numérico, variadores de velocidad, etc.

\section{Clase II:}

Sistemas mecánicos tradicionales con dispositivos electrónicos internos actualizados incorporados a los mismos, por ejemplo las modernas máquinas de coser.

\section{Clase III:}

Los sistemas que conservan la funcionalidad del sistema mecánico tradicional, solamente los mecanismos internos son sustituidos por la electrónica. Un ejemplo clásico es el reloj digital.

\section{Clase IV:}

Los productos diseñados con tecnologías mecánicas y electrónicas integrados sinérgicamente. Los ejemplos incluyen las fotocopiadoras, las lavadoras y secadoras, ollas automáticas, etc. (En esta clasificación se observa que el nivel de integración en el diseño de estos productos va evolucionando, gracias precisamente a la forma de trabajo que dio origen a la Mecatrónica, misma que también ha ido evolucionando)

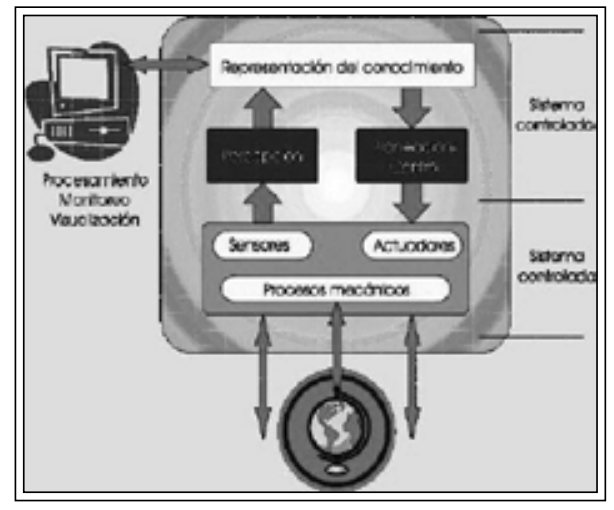

Figura 4. Arquitectura de un sistema Mecatrónico

En respuesta a ¿cómo diseñar el campo disciplinar?, la respuesta se encuentra presente, justamente en aplicar en la docencia de la mecatrónica: la 
cultura organizacional o filosofía de integración. Similar a la que produjo la interdisciplinariedad en el diseño de productos y sistemas Mecatrónicos (Aquino, Cuervo, Corona, (pp 141-145, 2011).

(Aquino, Corona Fernández, 2011), manifiestan que en la enseñanza de las ingenierías con una marcada interdisciplinariedad se deberán utilizar núcleos integradores y estos son precisamente aquellos que construyen la sinergia entre disciplinas, vea la Figura 5.

Sin embargo, por experiencia frente a grupos en nuestra unidad académica, hemos notado que con los núcleos integradores, no solo se construye la sinergia de tecnologías en los proyectos que se realizan, sino que, también a través de ellos se consolida el proceso enseñanza-aprendizaje de la práctica de la ingeniería, teniendo con ello el cumplimiento de los siguientes objetivos:

i. Dotar de utilidad práctica a los conocimientos científicos y tecnológicos que va adquiriendo de forma paralela a cada núcleo integrador.

ii. El alumno adquiere habilidades y la actitud propia de un ingeniero al utilizar en cada uno de ellos, de forma estratégica, el Método de la Ingeniería en la realización de cada proyecto en cada núcleo integrador.

iii. Todo eso que se describe en el perfil profesional de egreso, en los planes de estudios, se volverá una realidad con la integración de conocimientos y habilidades por medio de los núcleos integradores, ello debido al hecho, de que una formación integral en ingeniería requiere por necesidad, de una enseñanza integradora.

iv. Para el caso específico de ingenierías de corte interdisciplinar justamente con los núcleos se crea esa sinergia entre disciplinas, ya que mediante estos se establecen los vínculos que dan nombre (por ejemplo a la Ingeniería Biomédica, a la Ingeniería Mecatrónica, a la ingeniería Telemática, etc.); Razón de ser (soluciones que se ubican en la frontera de dos o más disciplinas y que solo se abordan con una visión conjunta e integradora) y razón de existir a dichas ingenierías, lo cual se explica en la razón evidente de que al resolver problemas tecnológicos, científicos y sociales, en donde se ha requerido de soluciones que involucran más de dos disciplinas científicas y/o tecnológicas; ellas son necesarias. Por ello, sí para la solución de tales problemas se han requerido de sinergia entre disciplinas es por demás lógico que para la enseñanza de ingenierías interdisciplinarias se requiera de una estrategia de enseñanza de ingeniería que integre de forma sinérgica tales disciplinas, vea figura 5, (Aquino, Corona, Cuervo, 2012b).

v. En el caso particular de la Ingeniería Mecatrónica, en el cursamiento de los núcleos integradores, se debe recrear la cultura organizacional que le dio origen y que la ha hecho evolucionar, provocando con ello que el egresado de esta rama de la ingeniería desarrolle las destrezas integrales y las habilidades de integración, que debe exhibir como profesional de ésta rama de la ingeniería en el sector productivo, observe la Figura 5. 
Aun cuando los planes y programas de estudio se pueden presentar bajo diversas formas, respondiendo a características diferentes de las diversas ingenierías. En Mecatrónica, se debe destacar, los que organizan los aprendizajes interdisciplinariamente, (por las razones que se explicaron en párrafos anteriores) siguiendo ciertos ejes (núcleos integradores) alrededor de los cuales orbitan los contenidos, en los cuales se vinculan la formación teórica y la formación en la práctica de la ingeniería. Estos núcleos forman al curriculum integrador, globalizador e interdisciplinar (Holístico). Los núcleos serán superadores de los límites de disciplinas, centrados en temas frontera que en el caso de la ingeniería implicara por fuerza una solución igualmente ubicada en la frontera entre disciplinas (Aquino, et al 2012b).

Esta proposición de integración y unidad está en oposición directa al paradigma de separación y fragmentación que predomina en el mundo contemporáneo, como se mencionó anteriormente.

Ahora bien dependiendo del desarrollo tecnológico, infraestructura y espacios, y aún más, de la disposición y de la visión que tengan los docentes de las escuelas que ofertan la enseñanza de la Mecatrónica. Las estrategias de enseñanza integradora podrían estar basadas en problemas, en proyectos o en casos. Sin embargo y aun con lo anterior, el nivel de integración que se alcance será directamente proporcional al número -y calidad-de núcleos integradores que haya en sus planes y programas de estudio.

Para ejecutar lo planteado se requiere concebir una forma de trabajo colegiado, muy similar a la cultura organizacional que provoco el origen de la Mecatrónica y que esta filosofía de trabajo tenga como plataforma una concepción integral en la formación de Ingenieros Mecatrónicos, (cosa que no es fácil de lograr). En ella deberán promoverse reuniones con temas precisos que orbiten alrededor de núcleos integradores en las que se promueva la participación sin excepción de ninguna índole de todos los profesores involucrados en la docencia de la Mecatrónica.

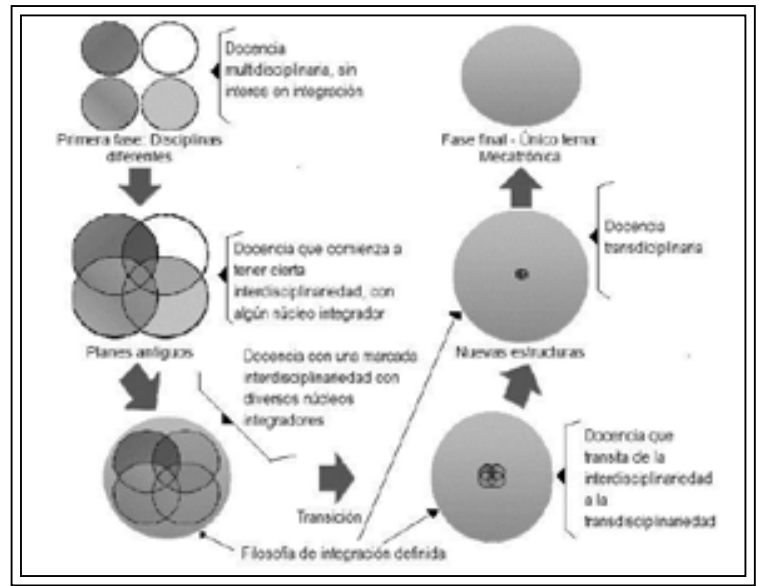

Figura 5. Evolución de la tendencia en la enseñanza de la Mecatrónica. 
El ejecutar proyectos que integren los conocimientos de diversas disciplinas y la práctica de la ingeniería, no es un una propuesta inédita de parte nuestra, actualmente la enseñanza de la ingeniería Mecatrónica en universidades de Colombia y de la ingeniería de telecomunicaciones en la Universidad Politécnica de Madrid se lleva a cabo ésta tarea, tal como se describe en Aquino R. et al (2010), logrando con ello, la enseñanza de la ingeniería en etapas o ciclos que al irlos cubriendo total o parcialmente, habilitaran al estudiante de ingeniería en destrezas bien definidas.

En la actualidad existen tantos modelos, criterios y currículos para formar un Ingeniero Mecatrónico, como universidades existen en el mundo (que ofrecen dicho programa). Con todo ello el perfil de egreso, cambia poco en el discurso o en el papel. Sin embargo, lo que sí es evidente en el campo disciplinar de cada universidad que ofrece este programa. Es el nivel de integración y la etapa en la que van transitando de acuerdo a la Figura 5. Por lo cual antes de definir un perfil de egreso, se deberá analizar en que etapa va transitando la enseñanza de la Mecatrónica en la escuela o facultad que la imparte.

Es necesario señalar que algunas escuelas, en la actualidad se imparte la Mecatrónica de forma multidisciplinar, otras escuelas más, ya han transitado a una enseñanza interdisciplinar, lo cual es evidente en su plan de estudios. Es recomendable en este tiempo para las escuelas que recién inician su impartición, comenzar ya con una enseñanza de corte interdisciplinar que posteriormente se transforme en una enseñanza transdiciplinar.

En ese contexto se puede señalar que la formación de un ingeniero Mecatrónico al igual que la Mecatrónica como rama de la ingeniería; tienen ambos una dimensión un tanto oculta, que es el nivel de integración en la enseñanza de la misma y la forma como se ejerce en la práctica finalmente. Esto mismo es evidente en la evolución descrita en la Figura 5.

\section{Conclusiones}

Actualmente se ha multiplicado la docencia de la Ingeniería Mecatrónica. Ésta ha experimentado un fuerte impulso, no solo en las naciones altamente desarrolladas, sino también en las naciones en vías de desarrollo. Sin embargo, se ha tomado poco cuidado en investigar la forma como la Ingeniería Mecatrónica fue concebida, al igual que tampoco se ha investigado la forma de recrear la cultura organizacional que la hizo emerger primeramente como concepto y que posteriormente la hizo crecer como rama interdisciplinar de la ingeniería.

Por estos hechos en este trabajo se han hecho evidentes los antecedentes y las preguntas que han llevado a mostrar los fundamentos necesarios para tratar de hacer realidad de una forma más eficiente y efectiva todo eso que se describe en el perfil 
de egreso del Ingeniero Mecatrónico en las escuelas de ingeniería, pero que en la práctica cuesta trabajo lograr. Y ello no por falta de profesionalismo, sino más bien por falta de conocimiento acerca de la evolución disciplinar de la Mecatrónica.

De igual forma es necesaria en la enseñanza de la Mecatrónica, recrear la forma de trabajo, filosofía o cultura organizacional que le dio origen y que deberá estar implícita en la forma de trabajo que cada egresado de esta rama de la ingeniería debe tener en el sector productivo.

Se ha dado una explicación de la evolución de la Mecatrónica y se ha pormenorizado el campo disciplinar de la misma. A fin de darle al lector una explicación clara de lo que es la Mecatrónica, ya con un corpus propio, autónomo y con una identidad diferenciada de las disciplinas que le sirvieron como fundamento.

Finalmente se proporciona una idea probada, para no solo crear la interdisciplinariedad, sino también para recrear la forma de trabajo, filosofía o cultura organizacional propia de la Mecatrónica, mediante los Núcleos Integradores. Los cuales no solo servirán para integrar disciplinas sino que deberán recrear la forma de trabajo que por excelencia tiene o debe tener aquel que haga Ingeniería Mecatrónica.

Estos núcleos integradores también serán los encargados de producir la transición de una Mecatrónica Interdisciplinaria a una Mecatrónica transdisciplinaria y finalmente conducir a la Mecatrónica a ser una rama de la Ingeniería y enseñada como tema único como hoy lo es la Ingeniería Eléctrica, la Ingeniería Mecánica o la Ingeniería Química. 


\section{Referencias}

Aquino J.A., Corona L. (2009) "La Ingeniería Mecatrónica en la Investigación, el desarrollo tecnológico y la innovación - Primera parte: Un modelo secuencial", Conversus, Revista de divulgación científica y tecnológica del Instituto Politécnico Nacional, México. No. 77 pp 29-31. ISSN-16652665

Aquino J.A V. D. Cuervo V.C., Corona L. (2011) "Construyendo un polo de innovación tecnológica a partir de un polo de innovación educativa". Revista de la Facultad de ingeniería de la Universidad Autónoma de Yucatán (UADY) No.2 2011-Nuevas tendencias en sistemas Mecatrónicos - pp 141-145 www.revista. ingenieria.uady.mx/volumen15/construyendo.pdf. ISSN: 1665-529X

Aquino Robles J.A. Corona L. (2009)“La Ingeniería Mecatrónica en la Investigación, el desarrollo tecnológico y la innovación - Segunda parte: Un modelo estratificado", Conversus, Revista de divulgación científica y tecnológica del Instituto Politécnico Nacional México No. 78 pp 28-31. ISSN-16652665

Álvarez Peña, C. Neff, F. J. Moya Rodríguez, J. L. Chagoyén Méndez, C. A. Machado Rodríguez, A. S. (2012) "Teaching Mechatronics engineering a challenge of the new century". Presentado en: The 2nd International Symposium on Integrating Research, Education, and Problem Solving. Orlando, Florida USA Aquino J.A., Corona L., Fernández C. (2009) "Impulsando el Desarrollo Sostenible, mediante Proyectos de Fin de Carrera en Ingeniería Mecatrónica, en nuestra Nación". $9^{\circ}$ Congreso de Internacional Retos y Expectativas de la Universidad organizado por Instituto Politécnico Nacional, México.

Aquino J.A., Fernández C., Cuervo V.D. (2010) Mecatrónicamente Sostenible. $8^{\circ}$. Congreso de Internacional sobre Innovación y Desarrollo Tecnológico CIINDET organizado por AMIME y la IEEE sección Morelos, sede Ciudad de Cuernavaca.

Aquino J.A., Corona L. Fernández C. (2011) "La Formación Integral en Ingeniería Mecatrónica" -. Presentado en el VI Congreso Internacional de Ingeniería Electromecánica y de Sistemas IPN- ESIME-SEPI México D.F.

Aquino J.A., Corona L., Fernández C., Cuervo V.D. (2010) "Trayectoria evolutiva de los criterios de evaluación en la enseñanza de la ingeniería Mecatrónica". IV Foro Nacional de Ciencias Básicas, organizado por la división de ciencias básicas de la Facultad de Ingeniería de la UNAM. México.

Aquino J.A., Corona L., Fernández C. (2012) "Identidad y destino del SER llamado Ingeniero marco histórico y referencial". Presentado en el Foro mundial 
de la enseñanza de la ingeniería, WEEF en Buenos Aires, Argentina.

Aquino J.A., Corona L., Cuervo V.D. (2012) "Creando el enlace entre las disciplinas de ciencias básicas en la enseñanza de la Ingeniería Mecatrónica”. V Foro Nacional de Ciencias Básicas, organizado por la división de ciencias básicas de la Facultad de Ingeniería de la Universidad Nacional Autónoma de México.

Aquino J.A., Corona L. Fernández C. (2012) "Sociedades estudiantiles en escuelas de ingeniería y la creación de conocimiento organizacional". Presentado en el Foro mundial de la enseñanza de la ingeniería, en Buenos Aires, Argentina.

Aquino J.A., Corona L., Fernández C. (2012) "La Mecatrónica Verde como actor y la lucha contra el cambio climático como escenario" Presentado en el $2^{\circ}$. Congreso Nacional en Investigación en Cambio Climático Organizado por la Universidad Nacional Autónoma de México.

Calderón E., Forero G. C.A., Chio N. (2011) “ La Enseñanza de la Ingeniería Mecatrónica en la UNAB y su metodología basada en Proyectos Integradores"; 3er Congreso Internacional de Ingeniería Mecatrónica - UNAB Vol 2, No 1. El código ISSN del evento es 2145-812X.

Castellanos Álvarez J., Lara Díaz L., Lapido Rodríguez M., Iglesias León M., Balbis Coll E., Navales Coll M., Álvarez González A. (2003) "Diseño Conceptual para la formación Holística del Ingeniero Mecánico". Biblioteca Virtual Universidad de Cienfuegos. Cuatro Caminos Km 4. Carreras a Rodas. Consultado en junio de 2013 en la web: http://www.bibliociencias.cu/gsdl/collect/eventos/ import/Formacion_holistica_ingeniero.pdf. 